Review began 10/07/2021 Review ended 11/22/2021 Published 11/25/2021

(c) Copyright 2021

Lum et al. This is an open access article distributed under the terms of the Creative Commons Attribution License CC-BY 4.0. which permits unrestricted use, distribution, and reproduction in any medium, provided the original author and source are credited.

\section{Emergency Pediatric Intubations in an Urban Children's Hospital Before and After Just-in-Time Training for Video Laryngoscopy}

Evan Lum ${ }^{1}$, Sherri Sommer-Candelario ${ }^{2}$, So Yung Choi ${ }^{3}$, Stephanie Delos Santos ${ }^{2}$, Kagen Aeby ${ }^{1}$, Jannet Lee-Jayaram 1,4

1. Pediatrics, University of Hawai'i, John A. Burns School of Medicine, Honolulu, USA 2. Pediatric Transport, Kapi'olani Medical Center for Women \& Children, Honolulu, USA 3. Quantitative Health Sciences, University of Hawai'i, John A. Burns School of Medicine, Honolulu, USA 4. Pediatrics, Kapi'olani Medical Center for Women \& Children, Honolulu, USA

Corresponding author: Jannet Lee-Jayaram, jannet98@hawaii.edu

\title{
Abstract
}

Objectives: The use of video laryngoscopy (VL) may augment emergency pediatric intubations outside the operating room (OR). Our objective was to describe the proportion of use and complications with VL before and after implementation of a VL just-in-time training (JITT).

Study design: This study was a retrospective chart review of pediatric intubations performed outside the OR at a single women and children's hospital from January 2015 to March 2020. Data were collected on patient age, intubation method, operator characteristics, adverse events, number of attempts, condition leading to intubation, and hospital location. Data were separated into pre-JITT (January 1, 2015 to April 31, 2018) and post-JITT (May 1, 2018 to March 1, 2020) periods. Descriptive statistics were used comparing pre- and postJITT periods for VL use, and the complications of intubations with multiple attempts (IMAs) and intubations with one or more adverse events (AEs).

Results: A total of 231 pediatric patients were intubated during the study period; 154 intubations in the preJITT and 77 intubations in the post-JITT periods. Pre- and post-JITT VL use was 17 (11\%) and 17 (22\%), respectively. With pre-JITT VL, there were four (23\%) IMAs and zero (0\%) intubation with one or more AE. With post-JITT VL, there were eight (47\%) IMAs and one (6\%) intubation with one or more AE.

Conclusion: The proportion of emergency pediatric intubations using VL increased after the institution of a JITT. There was no significant change in IMAs and AEs. The infrequency of pediatric intubations makes drawing conclusions regarding the impact on IMAs and AEs challenging. JITT may increase VL use for emergency pediatric intubations outside the OR and may be considered for refresher training, especially during the coronavirus disease 2019 (COVID-19) pandemic.

Categories: Emergency Medicine, Medical Simulation, Pediatrics

Keywords: laryngoscopy, healthcare simulation, just-in-time training, pediatric intubation, videolaryngoscopy

\section{Introduction}

Critically ill and injured pediatric patients frequently require intubation during the course of their medical care. Pediatric patients present additional challenges due to their anatomical differences that make intubation more difficult including smaller mouth, relatively larger tongue, larger occiput, floppier epiglottis, and narrow airway [1,2]. Additionally, pediatric patients have a higher metabolic rate, less reserve, and faster onset of hypoxia, making first-pass intubation success an even more critical goal than with adult patients $[1,2]$.

Video laryngoscopy (VL) is a method by which the glottic opening is visualized indirectly using a camera. The advantages of VL over direct laryngoscopy (DL) have been highlighted during the COVID-19 pandemic. Use of VL over DL for intubation is recommended to minimize risk by increasing first attempt success and distance from the patient's airway during this potentially aerosol-generating procedure $[3,4]$. While VL in adults may reduce the number of failed intubations, improve the glottic view, and reduce laryngeal trauma, in children, some evidence suggests that VL may lead to prolonged intubation with an increased rate of intubation failure when compared to DL $[5,6]$. VL has not demonstrated a consistent reduction in the number of intubation attempts nor the number of complications associated with intubation. Nevertheless, in light of the coronavirus disease 2019 (COVID-19) pandemic, the need to upskill medical providers in the use of VL has become evident.

Training methods for initial skill development and maintenance of skill retention vary. Just-in-time training (JITT), which originated in the automotive industry, is a planned, on-the-job, short unit of instruction 


\section{Cureus}

provided on-demand in close proximity to the need for the training [7]. JITT has demonstrated increased learner confidence in lumbar puncture, improved skill in VL and pediatric advanced life support, and decreased procedural skill decay in transvenous pacing catheter placement [8-11]. Its advantages include short time for training, in-situ location for training, and availability for repeated training.

JITT for VL was instituted at our tertiary care hospital in 2018 to upskill providers and staff in the use of this alternate method for visualizing the airway during intubations. The goal of this study was to describe the proportion of use and intubation complications with pediatric VL before and after implementation of VL JITT, prior to the COVID-19 pandemic. We excluded post-pandemic intubations in the expectation that the use of VL, the difficulty of intubations with additional personal protective equipment (PPE), and illness severity of patients would be affected by the COVID-19 pandemic.

\section{Materials And Methods}

A retrospective electronic chart review was performed of all intubations outside the OR at a single, urban, tertiary care, women and children's hospital in Honolulu, Hawaii from January 2015 (when VL was available at the institution) to March 2020 (when severe acute respiratory syndrome coronavirus 2 [SARS-CoV-2] infections were initially reported in Hawaii). The hospital is the only children's specialty hospital in the state, with the next closest pediatric hospital 2,600 miles away, and serves as the referral center for pediatric patients throughout the Pacific basin. Intubations outside the controlled OR environment were the focus, as these are generally emergent in nature, not performed by anesthesiologists, and high risk due to urgency and unknown fasting status. Intubation procedure notes were reviewed and data were collected on patient age, intubation method, provider specialty, adverse events, number of attempts, condition leading to intubation, and hospital location. In May 2018, we implemented a 15-minute VL JITT with hands-on simulated practice for emergency department (ED) and pediatric intensive care unit (PICU) providers and staff during regular work hours. JITT was provided by an ED attending physician and the transport nursing manager when workflow and patient census in the ED or PICU allowed. Participants included resident physicians, physicians, nurses, and respiratory therapists. All staff who care for critically ill patients outside the OR were invited to participate, even those who do not primarily perform intubations. This was to increase all staff's familiarity with equipment, including setting up, and how to best support the provider intubating, thereby increasing the likelihood of VL use. Participation was voluntary and repeatable. The JITT started with orientation to the equipment, then VL intubation demonstration by the instructor, and finally guided participant hands-on practice intubating an infant manikin. Equipment included a low-fidelity, intubatable, infant manikin, a VL (GlideScope ${ }^{\circledR}$, Verathon, Inc., Bothell, Washington), an endotracheal tube, and stylet (Figure 1).

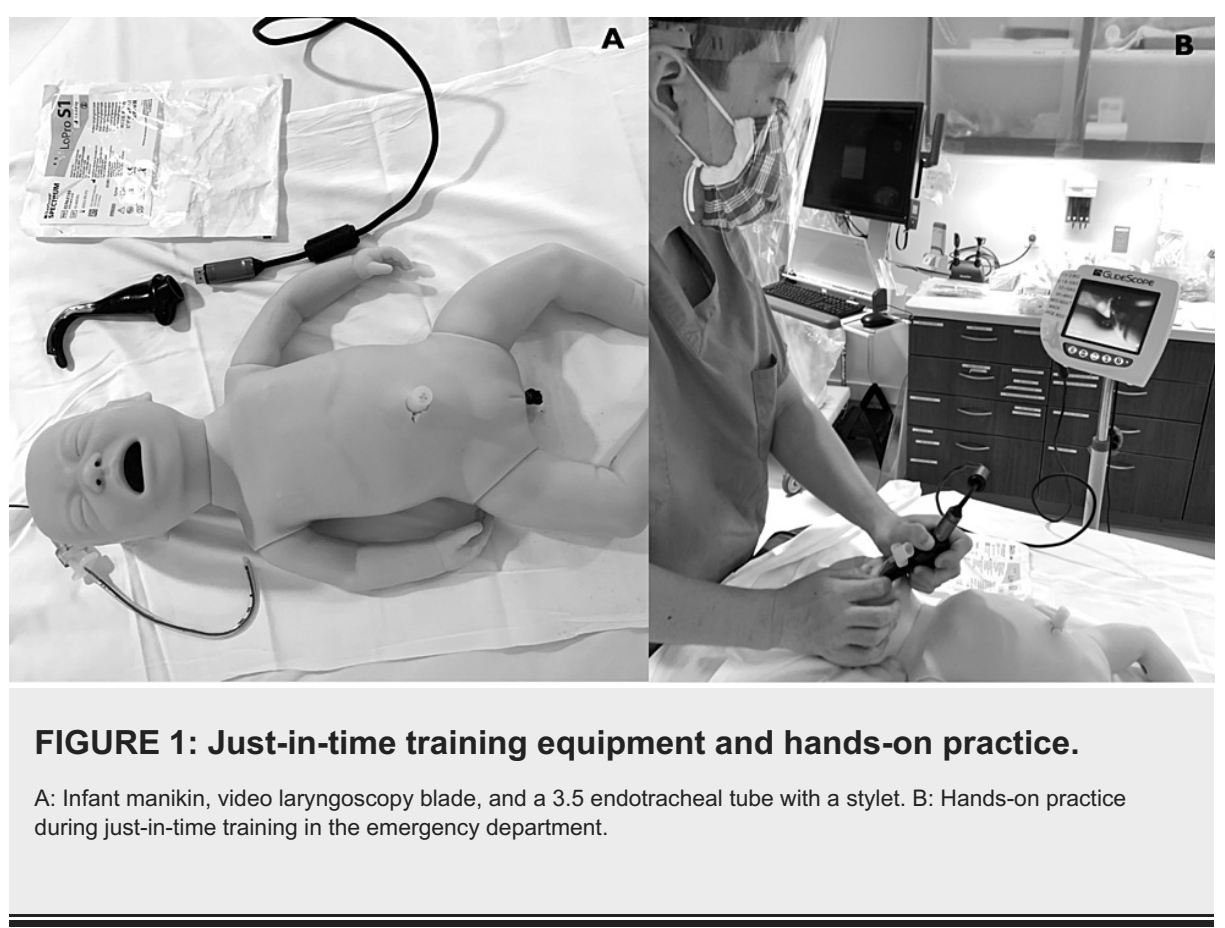

Pediatric intubation data were analyzed as training was focused on pediatric providers. Pediatric patients were defined as patients younger than 18 years old at the time of intubation. The data were separated into pre-JITT (January 1, 2015 to April 31, 2018) and post-JITT (May 1, 2018 to March 1, 2020) periods. The Institutional Official of Hawaii Pacific Health determined that the study was not a research subject to be reviewed by the Institutional Review Board. Descriptive statistics were used comparing pre- and post-JITT periods for VL use and the complications of (1) intubations with multiple attempts (IMAs) and (2) intubations with one or more adverse events (AEs). Change in VL use pre- and post-JITT was tested using 


\section{Cureus}

Fisher's exact test.

\section{Results}

A total of 268 patients were intubated outside the OR during the study period; 231 were pediatric patients. VL was used in 34 intubations and VL was not used in 197. There were no significant differences with respect to resident involvement, patient medical condition, and patient age $<1$ year (Table 1 ). There were 154 intubations in the pre-JITT and 77 intubations in the post-JITT period. There was a significant difference in pre- and post-JITT VL use, which was 17 (11\%) and $17(22 \%)$, respectively (p = 0.03, Figure 2). ED and PICU intubations were further analyzed as JITT was conducted in these units. Pre-JITT pediatric intubations in the ED and PICU numbered 72 (47\%) and 74 (48\%) with 12 (17\%) and five (7\%) intubations using VL. Post-JITT pediatric intubations in the ED and PICU numbered 32 (42\%) and 44 (57\%), with 14 (44\%) and three (7\%) intubations using VL (Tables 2, 3). With pre-JITT VL, there were four (23\%) IMAs and zero $(0 \%)$ intubation with one or more AE. With post-JITT VL, there were eight (47\%) IMAs and one $(6 \%)$ intubation with one or more $\mathrm{AE}$ (Table 4$)$.

\begin{tabular}{|c|c|c|c|}
\hline Characteristics & No VL $(n=197)$ & VL $(n=34)$ & p-value ${ }^{1}$ \\
\hline Intubation inserted by, $\mathbf{n}(\%)$ & & & 0.384 \\
\hline Non-resident or unknown & $177(90 \%)$ & $29(85 \%)$ & \\
\hline Resident & $20(10 \%)$ & $5(15 \%)$ & \\
\hline Condition, n (\%) & & & 0.111 \\
\hline Cardiovascular & $31(15.7 \%)$ & $3(8.8 \%)$ & \\
\hline Neurological & $64(32.5 \%)$ & $17(50.0 \%)$ & \\
\hline Respiratory & $57(28.9 \%)$ & $6(17.6 \%)$ & \\
\hline Sepsis/shock & $26(13.2 \%)$ & $2(5.9 \%)$ & \\
\hline Other/unknown & $19(9.6 \%)$ & $6(17.6 \%)$ & \\
\hline Age, n (\%) & & & 0.543 \\
\hline 0 year & $58(29 \%)$ & $8(23.5 \%)$ & \\
\hline $1+$ years & $139(71 \%)$ & $26(76.5 \%)$ & \\
\hline
\end{tabular}

TABLE 1: Characteristics of pediatric intubations $(n=231)$.

${ }^{1}$ Fisher's exact test; VL = video laryngoscopy . 


\section{Cureus}

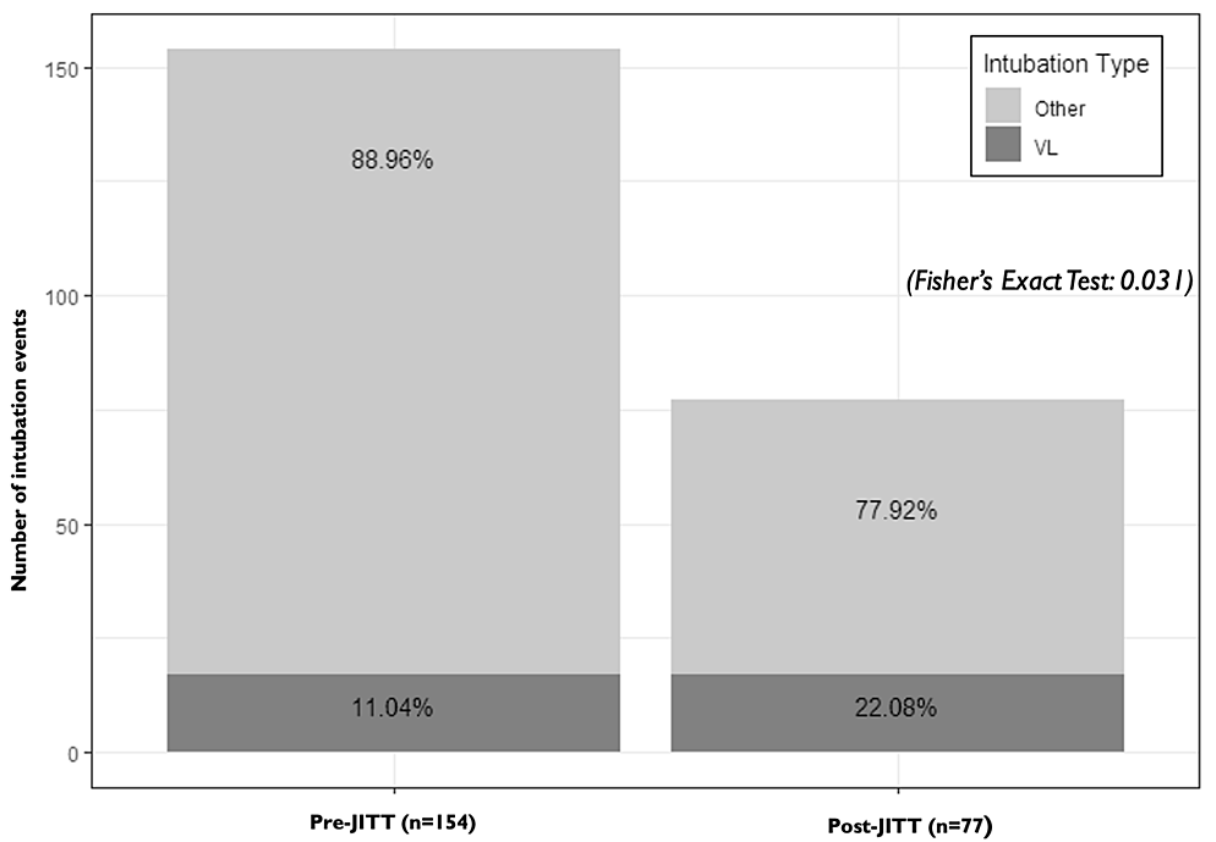

FIGURE 2: All intubations pre- and post-JITT.

Pre-JITT: 154 intubations; VL use was 17 (11\%). Post-JITT: 77 intubations; VL use 17 (22\%). There was increased video laryngoscopy use from the pre- to post-JITT periods $(p=0.03)$.

JITT, just-in-time training; VL = video laryngoscopy.

\begin{tabular}{|c|c|c|c|}
\hline & Pre $(n=72)$ & Post $(n=32)$ & p-value ${ }^{1}$ \\
\hline Other intubations & $60(83 \%)$ & $18(56 \%)$ & \multirow{2}{*}{0.006} \\
\hline VL intubations & $12(17 \%)$ & $14(44 \%)$ & \\
\hline
\end{tabular}

TABLE 2: Use of VL among ED patients $(n=104)$.

${ }^{1}$ Fisher's exact test; $\mathrm{VL}=$ video laryngoscopy; $E D=$ emergency department.

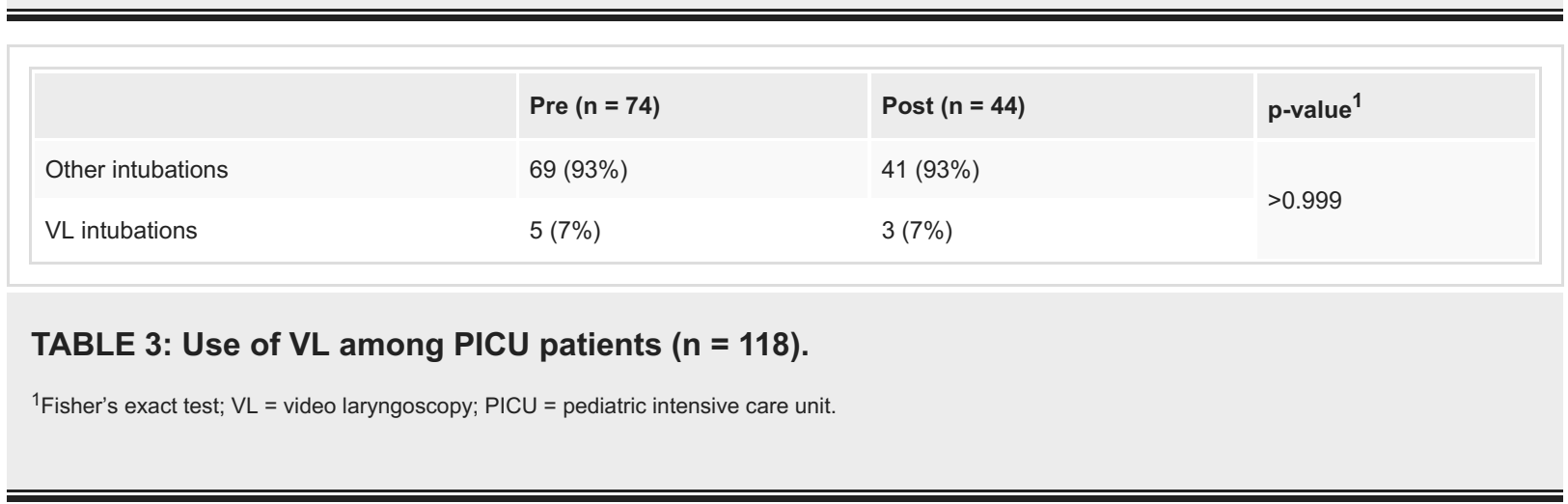




\section{Cureus}

\begin{tabular}{|c|c|c|c|}
\hline & Pre $(n=17)$ & Post $(n=17)$ & $p$-value ${ }^{1}$ \\
\hline Intubation attempts & & & 0.148 \\
\hline 1 & $6(35 \%)$ & $7(41 \%)$ & \\
\hline$\geq 2$ & $4(24 \%)$ & $8(47 \%)$ & \\
\hline Unknown & $7(41 \%)$ & $2(12 \%)$ & \\
\hline AEs & & & $>0.999$ \\
\hline None & $16(94 \%)$ & $15(88 \%)$ & \\
\hline 1 or more & $0(0.0 \%)$ & $1(6 \%)$ & \\
\hline Unknown & $1(6 \%)$ & $1(6 \%)$ & \\
\hline
\end{tabular}

TABLE 4: Pediatric patients with the use of VL $(n=34)$.

${ }^{1}$ Fisher's exact test; $\mathrm{VL}$ = video laryngoscopy; $\mathrm{AEs}=$ adverse events.

\section{Discussion}

VL use for pediatric intubations increased in the ED from the pre-JITT to the post-JITT period, but there was no significant change in the PICU. A systematic review and meta-analysis of adult and pediatric intubations outside the OR comparing VL to DL did not demonstrate any improvement in first-pass success in the ED with VL but did do so in the intensive care unit setting [12]. PICU intubations are more likely to be planned than ED intubations, after observation of clinical deterioration, which may influence the choice between DL and VL.

The infrequency of pediatric intubations with complications at this study's institution makes drawing significant conclusions regarding the impact on IMAs and AEs challenging. A 2016 report from the National Emergency Airway Registry of pediatric intubations in the ED analyzed 1,053 intubation events [13]. The first attempt's success rate was $83 \%$ and $\mathrm{AE}$ was noted in $15 \%$ of all encounters. Documentation standards for the participating centers were established by the registry allowing for more accurate quantification. Documentation at this study's institution was noted to be variable, with inconsistent reporting of intubation attempts and $\mathrm{AE}$, making accurate quantification difficult. In the 2016 report, VL was a predictor of first attempt success over DL among participating institutions that did not include any children's hospitals.

JITT at this study's institution demonstrated potential success in increasing VL use for emergency pediatric intubations outside the OR but a decrease in complications was not observed. The total number of complications may have been too low to detect any statistically significant changes. While a meta-analysis completed in 2017 on the use of VL in pediatric intubations did not demonstrate any advantage of using VL, a more recently published study, completed before the start of the pandemic, found VL was associated with higher first-attempt success over DL in pediatric ED patients [14].

Given that VL is recommended for all intubations during the COVID-19 pandemic, further intensive training may be required for providers participating in emergency pediatric intubations outside the OR in this new era. Reports after the start of the COVID-19 pandemic have confirmed that VL use has increased in emergency intubations $[15,16]$. Brief JITT for intubation using DL in a PICU did not improve resident first attempt or overall success but did increase resident participation in intubation events [17]. VL training using simulation-based mastery learning has demonstrated improved skill in pediatric residents when tested on manikins and a post-training first attempt success rate of $77.8 \%$, although pre-training first attempt success rate was not collected [18]. The resident training was conducted using mastery learning, requiring persistent training to a demonstrated competency level, and took between 30 minutes and two hours. While mastery learning is superior for skill acquisition in comparison to non-mastery simulation training, it also requires significantly more time [19]. As VL use increases by necessity, shorter, repeatable, JITT VL training may be more practical for busy pediatric healthcare providers.

In addition to necessity, there are other reasons that VL may be beneficial to use. During traditional DL, only the person intubating can visualize the airway, while the rest of the team is not able to determine the exact procedure step that is occurring. With VL, other providers and staff on the team can simultaneously visualize the airway and recordings can be preserved for future review. This concept of intubation sharing may be useful for coaching trainees, sharing information, research, and quality assurance [20]. In a training hospital, such as this study's institution, the use of VL may allow trainees safer intubation opportunities by direct visual supervision and coaching. Trainees who use a shared airway view with VL with their 
supervisors had higher intubation success rates [21]. They also reported that the teaching environment and constant guidance were helpful and improved their confidence [22].

There are several limitations to this study. The retrospective nature made accurate data collection challenging as intubation note detail varied between providers, including documentation of the number of attempts. JITT was performed with a convenience sample, during work hours, informally. As it was not tracked which providers participated in the JITT, it was not possible to determine if those who used VL were those who had JITT. The relatively small number of total intubations and subsequent AE made it challenging to draw specific conclusions, trends, and comparisons from the data. While VL use for pediatric intubations increased in the post-JITT period, causality cannot be attributed to the JITT as there may be other reasons use increased. However, the institution did have the VL device for three years and was not utilizing it with frequency, which was the occasion for the JITT implementation.

\section{Conclusions}

The proportion of emergency pediatric intubations using VL increased after the institution of a JITT, especially in the ED. There was no significant change in IMAs and AEs. The infrequency of pediatric intubations makes drawing conclusions regarding the impact on IMAs and AEs challenging. Continued refresher training, especially during the COVID-19 pandemic where increased VL use is expected, maybe important to upskill providers and staff in the use of VL.

\section{Additional Information \\ Disclosures}

Human subjects: Consent was obtained or waived by all participants in this study. Hawaii Pacific Health Research Institute issued approval 2018-043. On April 25, 2018, a designee of the Institutional Official of Hawaii Pacific Health determined the above-referenced study is not research (as defined in 45 CFR 46.102(d)) subject to be reviewed by an Institutional Review Board. The project was reviewed and determined to be a quality improvement activity and part of hospital operations as it seeks to improve patient care. Animal subjects: All authors have confirmed that this study did not involve animal subjects or tissue. Conflicts of interest: In compliance with the ICMJE uniform disclosure form, all authors declare the following: Payment/services info: So Yung Choi was partially supported by the Ola HAWAII (U54MD007601) grant from the National Institute of Health (NIH). The content is solely the responsibility of the authors and does not necessarily represent the official views of NIH. Financial relationships: All authors have declared that they have no financial relationships at present or within the previous three years with any organizations that might have an interest in the submitted work. Other relationships: All authors have declared that there are no other relationships or activities that could appear to have influenced the submitted work.

\section{Acknowledgements}

The project described was partially supported by the Office of the Dean through the Barry \& Virginia Weinman Endowment. So Yung Choi was partially supported by the Ola HAWAII (U54MD007601) grant from the National Institute of Health (NIH). The content is solely the responsibility of the authors and does not necessarily represent the official views of NIH.

\section{References}

1. Miller KA, Nagler J: Advances in emergent airway management in pediatrics . Emerg Med Clin North Am. 2019, 37:473-91. 10.1016/j.emc.2019.03.006

2. Cobb MJ: Just breathe: tips and highlights for managing pediatric respiratory distress and failure . Emerg Med Clin North Am. 2021, 39:493-508. 10.1016/j.emc.2021.04.004

3. Miller L, Luković E, Wagener G: Guiding airway management and personal protective equipment for COVID-19 intubation teams. Br J Anaesth. 2020, 125:e288-90. 10.1016/j.bja.2020.06.001

4. Cook TM, El-Boghdadly K, McGuire B, McNarry AF, Patel A, Higgs A: Consensus guidelines for managing the airway in patients with COVID-19: guidelines from the Difficult Airway Society, the Association of Anaesthetists, the Intensive Care Society, the Faculty of Intensive Care Medicine and the Royal College of Anaesthetists. Anaesthesia. 2020, 75:785-99. 10.1111/anae.15054

5. Lewis SR, Butler AR, Parker J, Cook TM, Smith AF: Videolaryngoscopy versus direct laryngoscopy for adult patients requiring tracheal intubation. Cochrane Database Syst Rev. 2016, 11:CD011136. 10.1002/14651858.CD011136.pub2

6. Abdelgadir IS, Phillips RS, Singh D, Moncreiff MP, Lumsden JL: Videolaryngoscopy versus direct laryngoscopy for tracheal intubation in children (excluding neonates). Cochrane Database Syst Rev. 2017, 5:CD011413. 10.1002/14651858.CD011413.pub2

7. Jones MJ: Just-in-time training. Adv Dev Hum Resour. 2001, 3:480-7. 10.1177/15234220122238409

8. Branzetti JB, Adedipe AA, Gittinger MJ, et al.: Randomised controlled trial to assess the effect of a just-intime training on procedural performance: a proof-of-concept study to address procedural skill decay. BMJ Qual Saf. 2017, 26:881-91. 10.1136/bmjqs-2017-006656

9. Grgurich E, Arnemann C, Amon K, Horton R, Carlson JN: Just-in-time video laryngoscopy versus direct laryngoscopy for neonatal intubation. J Perinat Neonatal Nurs. 2016, 30:367-71.

10.1097/JPN.0000000000000216 
10. Kamdar G, Kessler DO, Tilt L, et al.: Qualitative evaluation of just-in-time simulation-based learning: the learners' perspective. Simul Healthc. 2013, 8:43-8. 10.1097/SIH.0b013e31827861e8

11. Kurosawa H, Ikeyama T, Achuff P, et al.: A randomized, controlled trial of in situ pediatric advanced life support recertification ("pediatric advanced life support reconstructed") compared with standard pediatric advanced life support recertification for ICU frontline providers*. Crit Care Med. 2014, 42:610-8. 10.1097/CCM.0000000000000024

12. Arulkumaran N, Lowe J, Ions R, Mendoza M, Bennett V, Dunser MW: Videolaryngoscopy versus direct laryngoscopy for emergency orotracheal intubation outside the operating room: a systematic review and meta-analysis. Br J Anaesth. 2018, 120:712-24. 10.1016/j.bja.2017.12.041

13. Pallin DJ, Dwyer RC, Walls RM, Brown CA 3rd: Techniques and trends, success rates, and adverse events in emergency department pediatric intubations: a report from the National Emergency Airway Registry. Ann Emerg Med. 2016, 67:610-5. 10.1016/j.annemergmed.2015.12.006

14. Couto TB, Reis AG, Farhat SC, Carvalho VE, Schvartsman C: Changing the view: video versus direct laryngoscopy for intubation in the pediatric emergency department. Medicine (Baltimore). 2020, 99:e22289. 10.1097/MD.0000000000022289

15. Groombridge CJ, Maini A, Olaussen A, Kim Y, Fitzgerald M, Smit V: Unintended consequences: the impact of airway management modifications introduced in response to COVID-19 on intubations in a tertiary centre emergency department. Emerg Med Australas. 2021, 33:728-33. 10.1111/1742-6723.13809

16. Nauka PC, Chen JT, Shiloh AL, Eisen LA, Fein DG: Practice, outcomes, and complications of emergent endotracheal intubation by critical care practitioners during the COVID-19 pandemic. Chest. 2021, 3692:1113-2. 10.1016/j.chest.2021.06.008

17. Nishisaki A, Donoghue AJ, Colborn S, et al.: Effect of just-in-time simulation training on tracheal intubation procedure safety in the pediatric intensive care unit. Anesthesiology. 2010, 113:214-23. 10.1097/ALN.0b013e3181e19bf2

18. Couto TB, Reis AG, Farhat SC, Carvalho VE, Schvartsman C: Changing the view: impact of simulation-based mastery learning in pediatric tracheal intubation with videolaryngoscopy. J Pediatr (Rio J). 2021, 97:30-6. 10.1016/j.jped.2019.12.007

19. Cook DA, Brydges R, Zendejas B, Hamstra SJ, Hatala R: Mastery learning for health professionals using technology-enhanced simulation: a systematic review and meta-analysis. Acad Med. 2013, 88:1178-86. 10.1097/ACM.0b013e31829a365d

20. Miller KA, Marchese A, Luff D, Nagler J: Conceptualizing intubation sharing: a descriptive qualitative study of videolaryngoscopy for pediatric emergency airway management. AEM Educ Train. 2021, 5:e10589. 10.1002/aet2.10589

21. O'Shea JE, Thio M, Kamlin CO, et al.: Videolaryngoscopy to teach neonatal intubation: a randomized trial. Pediatrics. 2015, 136:912-9. 10.1542/peds.2015-1028

22. O'Shea JE, Kirolos S, Thio M, Kamlin CO, Davis PG: Neonatal videolaryngoscopy as a teaching aid: the trainees' perspective. Arch Dis Child Fetal Neonatal Ed. 2021, 106:168-71. 10.1136/archdischild-2020319619 